\title{
Tumor oncocítico hipersecretor de corteza suprarrenal, presentación de un caso
}

\section{Hypersecretor oncocytic tumor of adrenal cortex}

$1 \quad$ Mildrey García Hernández http://orcid.org/0000-0003-0497-7647 Hospital Universitario Comandante Faustino Pérez Hernández, Matanzas, Cuba mildreyg.mtz@infomed.sld.cu

$2 \quad$ Anairis Herrera Suárez http://orcid.org/0000-0003-1259-3491 Hospital Universitario Comandante Faustino Pérez Hernández, Matanzas, Cuba anairis@infomed.sld.cu

3 Luís Enrique Curbelo Gutiérrez $\quad$ (iD) http://orcid.org/0000-0002-7761-9595 Hospital Universitario Comandante Faustino Pérez Hernández, Matanzas, Cuba luis.curbelo@icloud.com

Artículo de Investigación Científica y Tecnológica Enviado: $11 / 11 / 2021$

Revisado: $26 / 11 / 2021$

Aceptado: 07/12/2021

Publicado:05/01/2022

DOI: https://doi.org/10.33262/anatomiadigital.v5i1.1971 Cítese: Digital, 5(1), 75-85. https://doi.org/10.33262/anatomiadigital.v5i1.1971

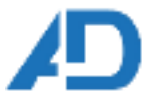

Ciencia Digital

ANATOMÍA DIGITAL, es una Revista Electónica, Trimestral, que se publicará en soporte electrónico tiene como misión contribuir a la formación de profesionales competentes con visión humanística y crítica que sean capaces de exponer sus resultados investigativos y científicos en la misma medida que se promueva mediante su intervención cambios positivos en la sociedad. https://anatomiadigital.org

La revista es editada por la Editorial Ciencia Digital (Editorial de prestigio registrada en la Cámara Ecuatoriana de Libro con No de Afiliación 663) www.celibro.org.ec

Esta revista está protegida bajo una licencia Creative Commons AttributionNonCommercialNoDerivatives 4.0 International. Copia de la licencia: http://creativecommons.org/licenses/by-nc-nd/4.0/ 
Palabras

claves: tumor adrenal, hipersecretor, oncocítico.

\section{Keywords:}

adrenal tumor, hypersecretor, oncocytic.

\section{Resumen}

Introducción: El carcinoma adrenocortical es una entidad rara con una incidencia anual de 1- 2 casos por millón de personas. Las neoplasias oncocíticas que surgen en las glándulas suprarrenales son extremadamente raras, y por lo general se descubren como tumores no funcionales y en su mayoría benignos. Objetivo: describir las características clínicoepidemiológicas del tumor oncocítico hipersecretor de corteza suprarrenal. Metodología: hicimos una revisión de la bibliografía sobre el tema y presentamos los resultados. Resultados: Desde la primera descripción confirmada por microscopía electrónica en 1986, se han notificado aproximadamente 147 casos en la literatura, con mayor frecuencia descritos como hallazgos incidentales. Se presenta el caso de una mujer con tumor secretor de corteza suprarrenal tratado exitosamente, con desaparición de la clínica inicial y que se mantiene con excelente calidad de vida. Conclusiones: se trata de una entidad rara cuyo manejo se basa fundamentalmente en la cirugía.

\section{Abstract}

Introduction: Adrenocortical carcinoma is a rare entity with an annual incidence of 1-2 cases per million people. Oncocytic neoplasms that arise in the adrenal glands are extremely rare, and are usually discovered as non- functional and mostly benign tumors. Objective: to describe the clinical- epidemiological characteristics of adrenal cortex secretor tumor. Methodology: we made a review of the bibliography on the subject and presented the results. Results: Since the first description confirmed by electron microscopy in 1986, approximately 147 cases have been reported in the literature, most often described as incidental findings. The case is presented of a woman with adrenal cortex secretor tumor successfully treated, with disappearance of the initial clinic and who remains with excellent quality of life. Conclusions: it is a rare entity whose management is mainly based on surgery. 


\section{Introducción.}

Los tumores suprarrenales son muy comunes, afectando entre el $3 \%$ y el $10 \%$ de la población humana, y la mayoría son pequeños adenomas adrenocorticales no funcionales benignos (ACA), el carcinoma adrenocortical (ACC), en cambio, es una enfermedad muy rara. La Oficina nacional de investigación de enfermedades raras de los Institutos Nacionales de Salud define las enfermedades raras por una prevalencia de menos de 200000 pacientes afectados en los Estados Unidos. Según esta definición, el ACC podría considerarse una enfermedad ultra rara. Se cree que la incidencia es de 1 a 2 por millón por año, pero aún faltan datos válidos. La base de datos de Vigilancia, Epidemiología y Resultados Finales (SEER) proporciona una estimación de la incidencia de aproximadamente 0,72 por millón de casos al año, lo que lleva al 0,2\% de todas las muertes por cáncer en los Estados Unidos. En el sur de Brasil, la incidencia es de 2,9 a 4,2 por millón al año.La mediana de edad para el diagnóstico es la quinta a la sexta década de vida, mientras que los registros alemanes informan una mediana de edad de 46 años. Tanto en el adulto como en la población pediátrica, existe una predilección por el género femenino (la proporción de mujeres a hombres oscila entre 1,5 y 2,5:1). Algunos estudios sugieren como factores de riesgo el tabaquismo en hombres y el uso de anticonceptivos en mujeres, especialmente antes de los 25 años. El papel de los estrógenos también ha sido sugerido por la observación de un probable aumento relativo del diagnóstico de ACC durante el embarazo. ${ }^{(1,2)}$

El carcinoma adrenocortical a pesar de ser una entidad poco frecuente, es el tipo más común de tumor canceroso de la glándula suprarrenal. El carcinoma adrenocortical puede ser un tumor funcional o no funcional. Si el tumor es funcional, puede producir hormonas que causan síntomas como presión arterial alta, nivel bajo de potasio, palpitaciones del corazón, nerviosismo, sentimientos de ansiedad o ataques de pánico, transpiración excesiva, diabetes, síndrome de Cushing, aumento de peso inexplicable o pérdida de peso, debilidad, estrías abdominales, crecimiento excesivo del cabello, cambios en los genitales, cambio en la libido, etc. Si el tumor no funciona, los síntomas ocurren porque el tumor ha crecido tanto que ejerce presión sobre los órganos cercanos, causando dolor abdominal o una sensación de saciedad. Para diagnosticar el carcinoma adrenocortical, además de un examen físico exhaustivo, se utilizan las siguientes pruebas: análisis de sangre y orina para ayudar a determinar si el tumor es funcional o no funcional, y pruebas de diagnóstico por imágenes (tomografía computarizada o resonancia magnética). El tratamiento depende de la etapa del cáncer. Se utilizan dos sistemas de estadificación principales: el sistema de ensayo TNM del Comité Conjunto Estadounidense contra el Cáncer (AJCC) y el sistema de puesta en escena ENSAT (Red Europea para el Estudio de los Tumores Suprarrenales). Ambos se basan en las mismas categorías de TNM. Por definición, el incidentaloma suprarrenal es una masa suprarrenal asintomática detectada en imágenes no realizadas para sospechas de 
enfermedad suprarrenal. En la mayoría de los casos, los incidentalomas suprarrenales son adenomas adrenocorticales no funcionales, pero también pueden representar afecciones que requieren intervención terapéutica (por ejemplo, carcinoma adrenocortical, feocromocitoma, adenoma productor de hormonas o metástasis). ${ }^{(3)}$

Si se diagnostica a tiempo, la resección quirúrgica completa +/- tratamiento adyuvante puede conducir a una supervivencia de 5 años de hasta el 80\%. Sin embargo, a menudo se diagnostica tarde y en la enfermedad avanzada, la supervivencia de 5 años es $<15 \%$ con una alta tasa de recurrencia incluso después de una cirugía radical. Los avances en el análisis genético en forma de secuenciación de próxima generación, ayudados por la bioinformática, han permitido la caracterización molecular de estos tumores. Esto, además de una mejor apreciación de los procesos de auto- renovación fisiológica y homeostática de la corteza suprarrenal, ha mejorado nuestra comprensión de la patogénesis de esta neoplasia maligna. La combinación de estos avances eventualmente conducirá al desarrollo de estrategias novedosas, eficaces y personalizadas. ${ }^{(4,5)}$

Los tumores suprarrenales malignos, que incluyen neuroblastoma, carcinoma adrenocortical con su variante oncocítica y una minoría de feocromocitomas suprarrenales, son extremadamente raros, con una incidencia de 1-2 por millón de habitantes anualmente. ${ }^{(6)}$

Las neoplasias oncocíticas u oncocitomas son en la mayoría de los casos tumores benignos, que generalmente surgen en los riñones o tiroides, paratiroides, glándulas salivales o pituitarias; rara vez, se han presentado en otros sitios incluyendo el tracto respiratorio (como carcinoma neuroendocrino oncocítico), laringe y plexo coroides. Las neoplasias oncocíticas que surgen en las glándulas suprarrenales son extremadamente raras, y por lo general se descubren como tumores no funcionales y en su mayoría benignos. Desde la primera descripción confirmada por microscopía electrónica en 1986, se han notificado aproximadamente 147 casos en la literatura, con mayor frecuencia descritos como hallazgos incidentales. A pesar del hecho de que tradicionalmente se les ha considerado tumores no funcionales y benignos, los datos recientes indican que alrededor del $20 \%$ de los oncocitomas adrenocorticales demuestran algunos elementos de la neoplasia maligna y entre el 10 y el $20 \%$ de ellos parecen afectar la producción de hormonas en las glándulas suprarrenales. teniendo en cuenta la rara incidencia de esta enfermedad, se llevó a cabo la siguiente presentación de caso con el objetivo de describir las características epidemiológicas, el comportamiento nosológico, los elementos diagnósticos y los principios básicos del manejo de esta entidad, mediante la presentación de caso tratado exitosamente. ${ }^{(7)}$ 


\section{Metodologia.}

Varias bases de datos de PubMed y la Biblioteca Nacional de Medicina fueron buscadas para estudios en todo el mundo que fueron publicados en inglés y traducidos al español en el período de 2015 a 2020. Después de examinar todos los resúmenes de las publicaciones identificadas por la búsqueda inicial, se incluyeron en el análisis estudios e informes de casos sobre pacientes con diagnóstico de tumores oncocíticos de corteza suprarrenal. La idoneidad de los estudios se definió a los efectos de esta revisión como la notificación de las características clínicas o biológicas, el tratamiento o los resultados clínicos de los pacientes con este diagnóstico.

\section{Resultados.}

Paciente blanca, femenina de 47 años que acudió con frecuencia a su médico del área de salud por presentar Hipertensión arterial y Diabetes Mellitus con cifras de Tensión Arterial y Glucemia que traducían descontrol metabólico a pesar de llevar tratamiento con 5 líneas terapéuticas e insulina a altas dosis respectivamente. Todo esto unido a una obesidad con apariencia de Síndrome de Cushing fue el motivo de ingreso para estudio diagnosticándose por imágenes la presencia de una masa tumoral en glándula suprarrenal derecha.

Fue operada el 4 de julio del 2019, se realizó excéresis de la lesión con margen oncológico y la biopsia informó: Carcinoma de corteza suprarrenal tipo oncocítico, de $8 \times 10 \times 4 \mathrm{cms}$, de alto grado, con extensas áreas de necrosis, numerosas mitosis atípicas que invaden la capsula, sin evidencia de invasión vascular. Luego de la cirugía la paciente evolucionó favorablemente, redujo su peso corporal, controló las cifras de TA y glucemia. Actualmente se encuentra asintomática, ECOG 0, con estudios de extensión negativos, controlada de las patologías antes mencionadas y en seguimiento.

Figura 1: Antes de la excéresis del tumor

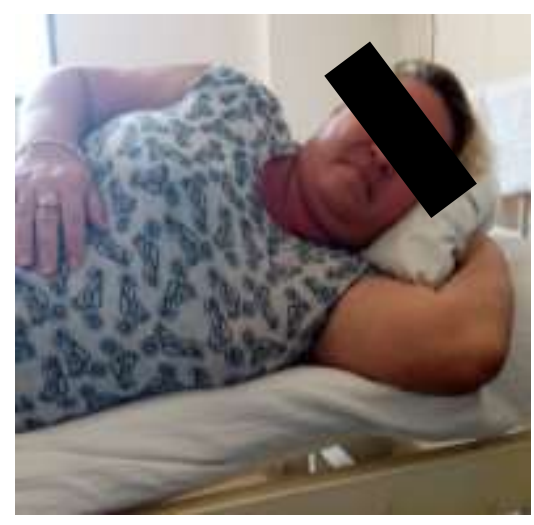

Fuente: Paciente
Figura 2: Luego del tratamiento quirúrgico

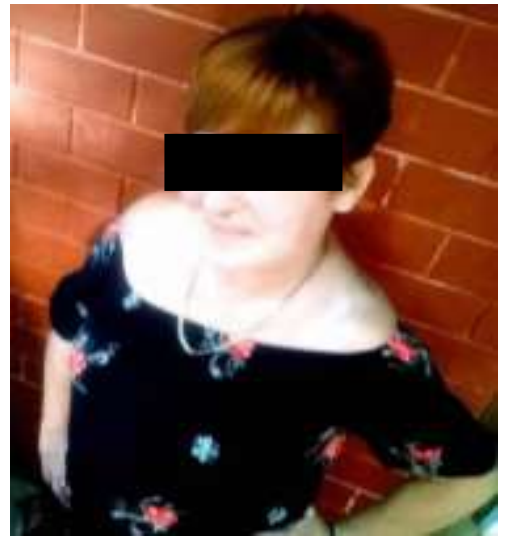

Fuente: Paciente 
Figura 3: Tumor resecado

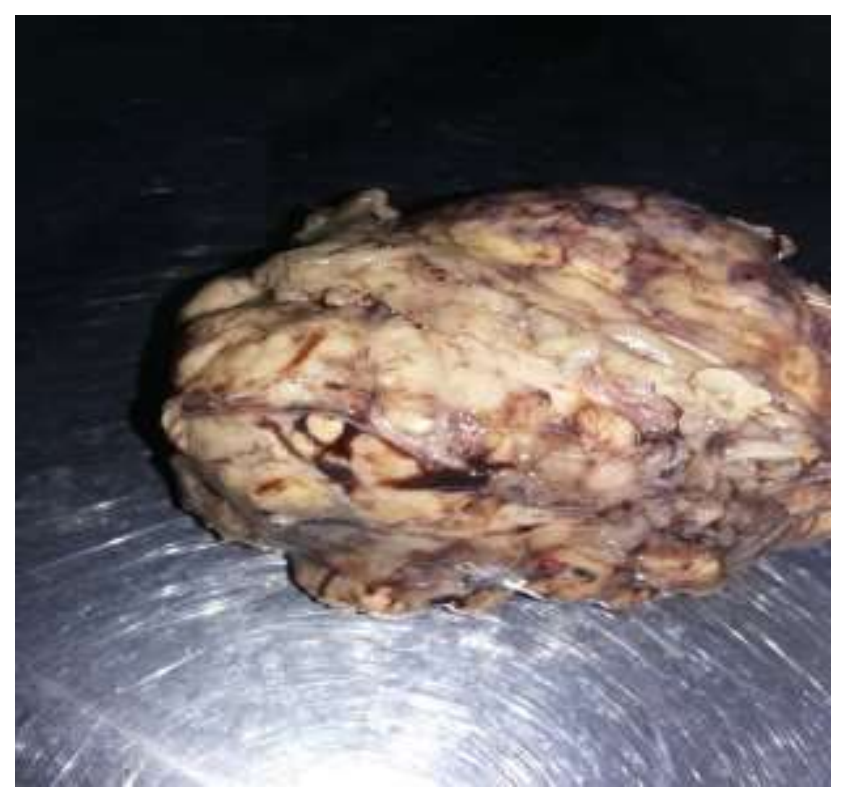

Fuente: Paciente

\section{Discusión}

El término 'oncocítico', deriva de la raíz griega 'onco-', que significa masa o volumen y utilizado por primera vez por Hamperl en 1950, por lo general describe una célula grande, altamente eosinofílica, granular, típicamente asociada con un tumor de células Hurthle de la glándula tiroides. También se conoce como una célula oxifílica (que se encuentra típicamente en las glándulas paratiroides) o una célula Askanazy (en la tiroides). ${ }^{(8)}$ El oncocito es de 1 a 2 veces el tamaño de las células normales que componen el tejido original, y por lo general muestra un citoplasma eosinofílico abundante y granular con un núcleo piknótico central. La granularidad citoplasmática se debe a la acumulación de mitocondrias que pueden ocupar hasta el 60\% del citoplasma. El aumento de la concentración de las mitocondrias se acompaña de una compresión gradual y a veces la desaparición de otros sistemas de membranas citoplasmáticas. La microscopía electrónica es una herramienta invaluable para la demostración de mitocondrias en el citoplasma de oncocitos. (9) La acidofilia característica debe distinguirse de la acidofilia que es secundaria a la acumulación de queratina, colágeno, lisosomas, gránulos neurosecretorios y citofilamentos, retículo endoplasmático liso o una combinación de estos. Las neoplasias oncocíticas suprarrenales tienen sus propias características estructurales. Macroscópicamente, la mayoría de ellos son una masa grande, redondeada, encapsulada y bien circunscrita, con un diámetro promedio de $8 \mathrm{~cm}$ $(2-20 \mathrm{~cm})$. La masa tiende a ser amarilla. Algunos tumores muestran áreas de hemorragia y necrosis. La clásica cicatriz radiante central que se ha descrito en la mayoría de los oncocitomas renales no siempre está presente en las neoplasias 
oncocíticas adrenocorticales. La apariencia microscópica de la neoplasia oncocítica incluye células dispuestas en patrones sólidos, trabeculares, tubulares o papilares. Las células tumorales son altamente eosinofílicas y granulares; rara vez, el oncocito tiene núcleos pleomórficos o una figura mitótica. ${ }^{(10)}$

El carcinoma cortical suprarrenal es una neoplasia maligna agresiva y, por lo general, anuncia un mal pronóstico. El subtipo oncocítico de esta neoplasia es poco frecuente, pero puede estar asociado con resultados más favorables. En uno de los estudios sobre esta entidad se realizó revisión exhaustiva de los gráficos y se recopilaron datos relacionados con la presentación, el tratamiento y los resultados. En el estudio, se identificaron 82 pacientes con carcinoma cortical suprarrenal. Se disponían de datos completos para 67 pacientes (82\%). En los 41 pacientes que se sometieron a resección, $9(22 \%)$ tenía subtipos oncocíticos. En comparación con el grupo total de carcinomas corticales suprarrenales, los subtipos oncocíticos fueron más grandes en la presentación $(19,8 \mathrm{~cm}$ vs $11,0 \mathrm{~cm})$, más comúnmente sintomáticos y hormonalmente activos, y a pesar de un mayor tamaño del tumor, fueron a menudo etapas tempranas I y II. Se observó enfermedad recurrente en 3 de 9 subtipos oncocíticos (frente a 23 de 32 carcinoma cortical suprarrenal), con mayor tiempo medio de recurrencia (17,5 vs 8 meses). ${ }^{(11,12)}$

En la mayoría de los casos, la neoplasia oncocítica suprarrenal se encuentra en la corteza suprarrenal, y solo se ha descrito 1 caso de un tumor oncocítico de la médula suprarrenal o en el tejido suprarrenal heterotópico. ${ }^{(13)}$

Hasta la fecha, no hay factores de riesgo específicos identificados (ambientales o genéticos), y hay un mínimo de conocimientos disponibles sobre los mecanismos que conducen a la oncocitosis en general y en las glándulas suprarrenales en particular. ${ }^{(14)}$ Estudios experimentales en ratas han demostrado que la $\mathrm{N}$-nitrosomorfina, un contaminante en el aire, induce oncocitosis renal y es seguido por la formación de oncocitoma renal, lo que sugiere que la proliferación mitocondrial excesiva es un mecanismo compensatorio en presencia de sustancias tóxicas. Otra hipótesis es que las neoplasias oncocíticas son tumores de las mitocondrias porque estos tienen su propio ADN que codifica sus propias proteínas características, y que el tumor surge a través de mutaciones en el genoma mitocondrial. Otra posible explicación es una diferenciación peculiar hacia las células de alta producción de energía o una respuesta fisiológica a un defecto en la maquinaria de producción de energía de la célula. ${ }^{(15)}$

Los dos factores más importantes en el diagnóstico de la masa suprarrenal, también válidos para la neoplasia oncocítica suprarrenal, son: el tamaño y/o la función de la lesión. El tamaño de una masa suprarrenal es muy importante en el proceso de toma de decisiones. En un informe en el que participaron 887 pacientes que tenían incidentalomas suprarrenales, se demostró que un diámetro superior a $4 \mathrm{~cm}$ tenía una 
sensibilidad del $90 \%$ para la detección del carcinoma adrenocortical, pero una especificidad baja; sólo el $24 \%$ de las lesiones fueron malignas. Las decisiones relativas a la cirugía también deben tener en cuenta el fenotipo de imagen de la masa, así como la edad del paciente y cualquier condición coexistente. El aumento de tamaño con el tiempo es otra indicación para la cirugía. En general, sin embargo, se recomienda la resección para todas las masas suprarrenales de $16 \mathrm{~cm}$. El tamaño es un problema en las neoplasias oncocíticas suprarrenales porque la mayoría de los tumores suprarrenales oncocíticos, aunque benignos, muestran un gran volumen. ${ }^{(16)}$

Se deben realizar análisis bioquímicos cuidadosos para descartar la presencia de una masa de funcionamiento primario que requiere resección quirúrgica a pesar del tamaño: algunos casos de neoplasia oncocítica suprarrenal se han presentado como tumores en funcionamiento. ${ }^{(17)}$ Es extremadamente raro encontrar un caso de neoplasia oncocítica suprarrenal con una presentación clínica que imita el feocromocitoma, un hallazgo que plantea un dilema diagnóstico preoperatorio considerable, ya que el propio feocromocitoma puede confundirse con neoplasia oncocítica. La distinción es posible en la mayoría de los casos, basada en estudios clínicos, bioquímicos e inmunohistoquímicos. En raras ocasiones, el síndrome de Cushing es causado por un subtipo oncocítico de carcinoma adrenocortical maligno. ${ }^{(18,19)}$

El tratamiento de la masa suprarrenal depende del tamaño y la función. El manejo quirúrgico de tumores suprarrenales oncocíticos ha sido tradicionalmente un enfoque quirúrgico abierto. La adrenalectomía es el estándar, ya que la neoplasia oncocítica suprarrenal generalmente se presenta como gran masa suprarrenal. En algunos casos se propone una adrenalectomía parcial. ${ }^{(20,21)}$

\section{Conclusiones.}

- Los tumores oncocíticos de corteza suprarrenal son raros y por lo general se descubren como tumores no funcionales y en su mayoría benignos.

- Se trata de una entidad muy sintomática, caracterizada por la secreción activa de hormonas suprarrenales.

- El tratamiento fundamental es la cirugía y el pronóstico es generalmente malo.

\section{Referencias bibliográficas.}

Else T, Kim AC, Sabolch A, Raymond VM, Kandathil A, Caoili EM, et al. Adrenocortical carcinoma [Internet]. Vol. 35, Endocrine Reviews. Endocrine Society; 2014 [citado 19 de diciembre de 2020]. p. 282-326. Disponible en: https://pubmed.ncbi.nlm.nih.gov/24423978/ 
Lam AK yin. Update on Adrenal Tumours in 2017 World Health Organization (WHO) of Endocrine Tumours [Internet]. Vol. 28, Endocrine Pathology. Humana Press Inc.; 2017 [citado 20 de diciembre de 2020]. p. 213-27. Disponible en: https://pubmed.ncbi.nlm.nih.gov/28477311/

Kranjčević K. [ADRENOCORTICAL CARCINOMA]. Acta Med Croatica. diciembre de 2016;70(4-5):315-8.

Pittaway JFH, Guasti L. Pathobiology and genetics of adrenocortical carcinoma [Internet]. Vol. 62, Journal of Molecular Endocrinology. BioScientifica Ltd.; 2019 [citado 19 de diciembre de 2020]. p. R105-19. Disponible en: https://pubmed.ncbi.nlm.nih.gov/30072419/

Hodgson A, Pakbaz S, Mete O. A Diagnostic Approach to Adrenocortical Tumors [Internet]. Vol. 12, Surgical Pathology Clinics. W.B. Saunders; 2019 [citado 20 de diciembre de 2020]. p. 967-95. Disponible en: https://pubmed.ncbi.nlm.nih.gov/31672302/

de Krijger RR, Papathomas TG: Adrenocor-tica 1 neoplasia: evolv ing concepts in tumori-genesis with an emphasis on adrenal cortical carcinoma variants. Virchows Arch 2012; 460: 9-18.

Sav A, Scheithauer BW, Mazzola CA, Ketter-ling SR, Thompson SJ, Reilly MH: Oncocytic choroid plexus carcinoma: case report. Clin Neuropathol 2010; 29: 14 -20 .

Hamperl H: Oncocytes and the so-called Hurthle cell tumor. Arch Pathol 1950; 49: $563-570$.

Mearini L, Del Sordo R, Costantini E, Nunzi E, Porena M. Adrenal oncocytic neoplasm: A systematic review [Internet]. Vol. 91, Urologia Internationalis. Urol Int; 2013 [citado 20 de diciembre de 2020]. p. 125-33. Disponible en: https://pubmed.ncbi.nlm.nih.gov/23147196/

Seo IS, Henley JD, Min KW: Peculiar cyto-plasmic inclusions in oncocytic adrenal cortical tumors: an electron microscopic observation. Ultrastruct Pathol 2002; 26: $229-235$.

Mills JK, Khalil M, Pasieka J, Kong S, Xu Y, Harvey A. Oncocytic subtypes of adrenal cortical carcinoma: Aggressive in appearance yet more indolent in behavior? En: Surgery (United States) [Internet]. Mosby Inc.; 2019 [citado 19 de diciembre de 2020]. p. 524-33. Disponible en: https://pubmed.ncbi.nlm.nih.gov/31472975/ 
Ertan Y, Argon A, Özdemir M, Yürekli BPŞ, Dökümcü Z, Makayc Ö. Oncocytic adreno cortical tumors: Pathological features of 16 cases and review of the literature [Internet]. Vol. 36, Journal of Environmental Pathology, Toxicology and Oncology. Begell House Inc.; 2017 [citado 20 de diciembre de 2020]. p. 237-44. Disponible en: https://pubmed.ncbi.nlm.nih.gov/29283337/

Corsi A, Riminucci M, Petrozza V, Collins MT, Natale ME, Cancrini A, Bianco P: Inci-dentally detected giant oncocytoma arising in retroperitoneal heterotopic adrenal tissue. Arch Pathol Lab Med 2002; 126: 1118 -1122 .

Bonnet-Serrano F, Bertherat J. Genetics of tumors of the adrenal cortex [Internet]. Vol. 25, Endocrine-Related Cancer. BioScientifica Ltd.; 2018 [citado 20 de diciembre de 2020]. p. R131-52. Disponible en: https://pubmed.ncbi.nlm.nih.gov/29233839/

Duregon E, Volante M, Cappia S, Cuccurullo A, Bisceglia M, Wong DD, Spagnolo DV,Szpak-Ulczok S, Bollito E, Daffara F, Berruti A, Terzolo M, Papotti M: Oncocytic adreno-cortical tumors: diagnostic algorithm and mitochondrial DNA profile in 27 cases. Am J S.

Angeli A, Osella G, Ali A, Terzolo M: Adre-nal incidentaloma: an overview of clinical and epidemiological data from the National Italian Study Group. Horm Res 1997; 47: 279-283.

Carré J, Grunenwald S, Vezzosi D, Mazerolles C, Bennet A, Meduri G, et al. Virilizing oncocytic adrenocortical carcinoma: clinical and immunohistochemical studies. Gynecol Endocrinol [Internet]. 2 de agosto de 2016 [citado 20 de diciembre de 2020];32(8):662-6. Disponible en: https://pubmed.ncbi.nlm.nih.gov/26954035/

Goel T, Thomas J, Garg S, Rao AC, ReddyS: Adrenal oncocytoma masquerading as a functional tumor. Indian J Urol 2007; 23: 77-78.ddyS: Adrenal oncocytoma masquerading as a functional tumor. Indian J Urol 2007; 23: 77-78.

Li M, Wenig BM: Adrenal oncocytic pheo-chromocytoma. Am J Surg Pathol 2000; 24: $1552-1557$.

Tian XJ, Huang Y, Lu J, Ma LL: Laparoscopic adrenocortical oncocytoma resection. Chin Med J 2008; 121: 862-864.

Erickson LA. Challenges in surgical pathology of adrenocortical tumours [Internet]. Vol. 72, Histopathology. Blackwell Publishing Ltd; 2018 [citado 20 de diciembre de 2020]. p. 82-96. Disponible en: https://pubmed.ncbi.nlm.nih.gov/29239032/ 
El artículo que se publica es de exclusiva responsabilidad de los autores y no necesariamente reflejan el pensamiento de la Revista Anatomía Digital.

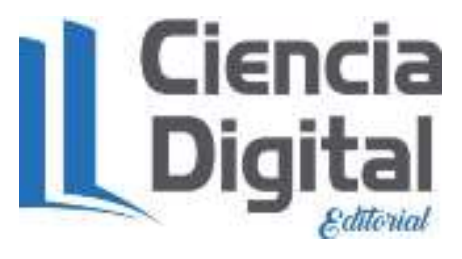

El artículo queda en propiedad de la revista y, por tanto, su publicación parcial y/o total en otro medio tiene que ser autorizado por el director de la Revista Anatomía Digital.
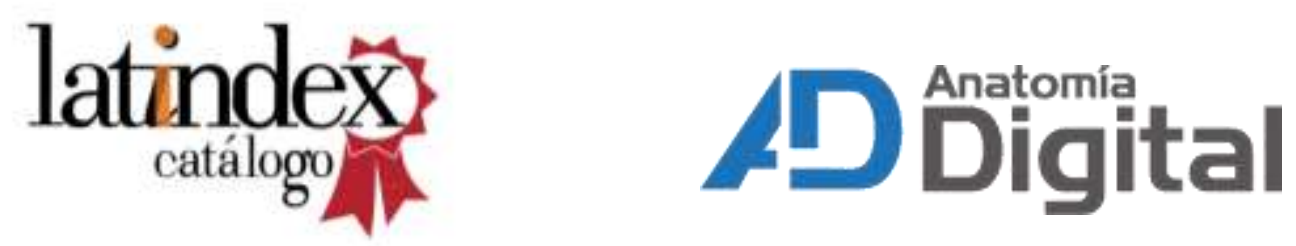

Indexaciones

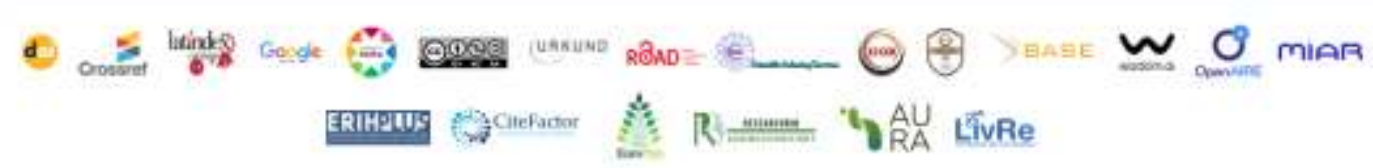

